\title{
Routine screening for sleep apnoea by dentists now recommended
}

Dentists interested in sleep medicine can play a more extensive role in diagnosing and treating patients who suffer from sleep-related problems, according to Professor Ama Johal, the British Orthodontic Society (BOS) authority on dental sleep medicine and Vice-President of the British Society of Dental Sleep Medicine.

Professor Ama Johal (pictured) explained that sleep-related breathing disorders can range from 'simple' snoring to obstructive sleep apnoea (OSA), a serious medical condition characterised by repeated collapse of the pharyngeal airway at night, leading to sleep deprivation. Exhaustion is not the only side effect. There is evidence that sleeplessness can act as an independent risk factor for Type II diabetes and cardiovascular disease. OSA is associated with a significant cardiovascular and metabolic co-morbidity, impaired quality of life, and increased risk of car accidents.

Professor Johal said: 'Managing patients with OSA is very much the domain of multi-disciplinary clinics, within which the dental profession now has an established role. We regularly see patients with more serious sleep-related problems but where the problems are milder, colleagues in general dental practice can manage their treatment'.

Professor Johal says that there is now a clearer diagnostic and referral pathway with improved techniques for treating patients who have mildmoderate sleep apnoea or snoring problems. He is encouraging dentists to screen for sleep-related problems and OSA as a matter of routine.
For those with symptomatic OSA, the first line therapy is continuous positive airway pressure (CPAP) in view of its proven treatment effect. This involves the patient wearing a mask attached to a pump, which filters, humidifies and directs air under pressure into the upper airway, in an attempt to prevent its collapse throughout the night.

However, the limitations of CPAP are compliance, which can be very poor, and in some patients may not resolve the OSA. However, there have been improvements in mandibular advancement appliances (MAA), a custom-made mouthguard, which works by posturing the lower jaw forward, keeping the airway open.

The advantage of the latest design of MAA is that they permit the mandible to be advanced in a step-wise manner, so the amount of advancement can be tailored to the specific needs of the individual. This has been scientifically demonstrated as being important and beneficial.

Appliances which permit the patient to adjust the jaw protrusion in small progressive movements $(0.5 \mathrm{~mm})$ allow for a precise effective point to be determined, referred to as 'mandibular titration'. The benefits are that patients can then adapt at a more gradual rate to their appliance, which in turn can improve their acceptance, helping to achieve the high rates of compliance achieved with these devices. Furthermore, unwanted tooth movement which can be caused by MAAs is minimised.

\section{BOOK REVIEW}

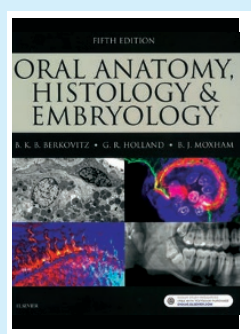

ORAL ANATOMY, HISTOLOGY AND EMBRYOLOGY, FIFTH EDITION

Barry Berkovitz G. Holland Bernard Moxham

Elsevier; 2017

price $\mathrm{f64.99}$ pp. 472

ISBN: 9780723438120

The fifth edition of this classic textbook Oral anatomy, histology and embryology presents readers with an in-depth understanding of the essential subject matter relevant to dental practice. This latest edition equips both dental undergraduates and postgraduates with the most recent research, in a common atlas-style format. This book is especially useful for those preparing for MJDF, MFDS or ORE examinations.

Structural updates to the edition include 'Learning objectives' to help students focus in on critical areas, 'Overviews' at the start of chapters to help place content into broader context, updated artwork, enlargement and standardisation of tooth and pulp morphology photographs.

Chapters have also been reworked to cover functional and radiological anatomy, enamel, alveolar bone, the salivary glands, the temporomandibular joint, amelogenesis and dentinogenesis. The book is formatted like other Elsevier textbooks such as the renowned Scully's handbook of medical problems in dentistry. There are 27 logically sequenced chapters. It begins with the appearance of the oral cavity (chapter 1), then giving overviews of different area anatomy of the head and neck. Useful in these sections is the 'Clinical considerations' which bring to life the practical applications of the detailed anatomy that general practitioners will appreciate.

The middle part of the book sheds more light on structures of the oral cavity such as enamel, dental pulp and salivary glands. The final part of the book deals with developmental processes of the face, jaw, early tooth development and other areas of significance. The final chapter may act as a bonus for some readers, giving insights into the ageing of dental tissue, dental archaeology and anthropological applications of tooth structure.

The authors must be congratulated for making the difficult topic of head and neck embryology easier to understand for dental students using clear diagrams, photographs and explanations. The clarity of this book will attract students to become deep learners of the subject matter, able to take this knowledge beyond exams to improve their clinical practice and/or their academic rigour.

Overall, this excellent, yet hefty textbook, big enough to sit on some dental coffee tables, provides a wealth of information to curious students. In many respects, it offers critical 'core knowledge' every dental practitioner should be aware of. Although its size does not allow it to be carried at leisure, it comes with an online programme to help readers mobilise their learning. The updated version could add patient case studies relevant to the academic knowledge to the 'Clinical considerations' sections.

Vinay Raniga 